\title{
FUNCIONALIDAD Y CONVIVENCIA DEL ESPAÑOL Y EL VASCO EN EL PAISAJE LINGÜíSTICO DE BILBAO
}

\author{
Functionality and Coexistence of Spanish and Basque in Bilbao's linguistic \\ LANDSCAPE
}

Fonctionnalité et COexistence de l'espagnol et du basQue dans Le Paysage LINGUISTIQUE DE BILBAO

\author{
Carmen Fernández Juncal \\ Ph. D. en Lengua Española, \\ Universidad de Salamanca, España. \\ Profesora titular del Departamento \\ de Lengua Española, Universidad de \\ Salamanca, España. \\ cjuncal@usal.es \\ http://orcid. \\ org/0000-0002-7015-4983
}

\begin{abstract}
RESUMEN
El español y el vasco conviven desde siglos atrás con diferentes grados de permeabilidad y complementariedad, dependiendo del entorno físico y temporal en el que se desarrolla esa coexistencia. El presente estudio, de carácter mixto, cuantitativo y cualitativo, examina el actual paisaje lingüístico de Bilbao, para determinar las diferentes funciones que asumen las dos lenguas en un territorio donde un uso mayoritario del español alterna con una política muy activa de promoción del vasco. A partir de un corpus de imágenes con textos públicos, se llevó a cabo un proceso de clasificación de los signos lingüísticos de acuerdo con diversos parámetros. El análisis cuantitativo resalta la presencia minoritaria del vasco respecto del español, como reflejo del empleo de esas dos variedades en esa misma área. También se deduce un reparto de funciones entre ambas: mayor función informativa-denotativa para el español y mayor función simbólicaconnotativa para el vasco. Esta situación hace reconsiderar los papeles tradicionales entre lenguas mayoritarias y lenguas minoritarias.
\end{abstract}

Palabras clave: paisaje lingüístico; vasco; español; Bilbao; funcionalidad; coexistencia lingüística.

\begin{abstract}
Spanish and Basque have lived together for centuries with different degrees of permeability and complementarity depending on the physical and temporal environment in which this coexistence takes place. The present study, of a mixed, quantitative and qualitative nature, examines the current linguistic landscape of Bilbao in order to determine the different functions assumed by Spanish and Basque in a territory where majoritary use of Spanish alternates with a very active policy of promoting Basque. Based on a corpus of images with public texts, a process of classification of the linguistic signs according to different parameters was carried out. The quantitative analysis highlights the minoritary presence of Basque in relation to Spanish, as a reflection of the use of these two varieties in the same area. There is also a division of functions between the two: greater informative-denotative function for Spanish and greater symbolic-connotative function
\end{abstract}


for Basque. This situation calls for a reconsideration of the traditional roles between majority languages and minority languages.

Keywords: Linguistic landscape; Basque; Spanish; Bilbao; functionality; linguistic coexistence.

\section{RÉSUMÉ}

Espagnol et Basque vivent ensemble depuis des siècles avec des degrés de perméabilité et de complémentarité différents selon l'environnement physique et temporel dans lequel cette coexistence a lieu. Cette étude, de nature mixte, quantitative et qualitative, examine le paysage linguistique actuel de Bilbao afin de déterminer les différentes fonctions assumées par l'espagnol et le basque dans un territoire où l'utilisation majoritaire de l'espagnol alterne avec une politique très active de promotion du basque. Sur la base d'un corpus d'images avec des textes publics, un processus de classification des signes linguistiques a été réalisé selon différents paramètres. L'analyse quantitative met en évidence la présence minoritaire du basque par rapport à l'espagnol, reflet de l'utilisation de ces deux variétés dans la même zone. Il y a aussi une répartition des fonctions entre les deux : plus grande fonction informative et dénotative pour l'espagnol et plus grande fonction symbolique et connotative pour le basque. Cette situation nous amène à reconsidérer les rôles traditionnels entre les langues majoritaires et les langues minoritaires.

Mots clés : paysage linguistique ; basque ; espagnol ; Bilbao ; fonctionnalité ; coexistence linguistique. 


\section{Introducción}

El presente trabajo centra su atención en la convivencia y distribución de funciones que se lleva a cabo entre la lengua española y la lengua vasca en el paisaje lingüístico de Bilbao, dentro de una línea de investigación que ha demostrado ser muy fructífera durante las últimas décadas.

Aunque con anterioridad se habían realizado estudios en torno al discurso escrito en el ámbito público (Spolsky, 2009), es el trabajo de Landry y Bourhis (1997) el que marca el comienzo de las investigaciones sobre el paisaje lingüistico ( $\mathrm{PL}$ ). Además de crear el término técnico que designa el campo de acción de una emergente disciplina, estos autores aportan una definición que acota esa tarea:

[...] el lenguaje de las señales de tráfico, los carteles publicitarios, los nombres de las calles, los nombres de los lugares, los carteles de las tiendas comerciales y los letreros públicos de los edificios gubernamentales se combinan para formar el paisaje lingüístico de un determinado territorio, región o aglomeración urbana (Landry y Bourhis, 1997, p. 25). ${ }^{1}$

Sin embargo, a efectos de nuestra investigación, lo más significativo es que los mismos autores (1997, pp. 25-29) adscriben a este PL dos funciones principales: una informativa y una simbólica. Mediante la primera, el PL constituye un marcador distintivo del territorio geográfico habitado por una comunidad lingüística. Simultáneamente, el PL permite delinear los límites de una comunidad lingüística respecto a otras e informar a sus miembros (o a personas pertenecientes a otra comunidad) del perfil lingüístico y las fronteras de la región, por lo que se crean expectativas sobre la herramienta de intercambio que se empleará en esa zona (aunque no siempre las expectativas se cumplen, sobre todo en entornos multilingües).

Habida cuenta de que el plurilingüismo es un fenómeno común a las sociedades modernas, esta faceta informativa del PL documenta también sobre los

1 Las traducciones del inglés son de la autora. grupos que conviven en esa sociedad, reflejando así la diversidad, en mayor o menor grado, que la caracteriza, y sobre las relaciones de poder que se establecen entre las distintas variedades, lo que incluye la política lingüística, existente o no, explícita o implícita, que pudiera estar implementada en la comunidad. Es decir, sin tener que pronunciar una simple palabra, el PL provee datos al recién llegado acerca de los usos lingüísticos del área, la posibilidad o no de comunicarse, y de tener servicios en su propia lengua (Landry y Bourhis, 1997, p. 25).

Por su parte, la función simbólica alude a la sensación de inclusión o exclusión que provoca el PL en un entorno bilingüe o multilingüe en sus usuarios, en especial en aquellos hablantes que pertenecen a un grupo lingüístico minoritario, cuya variedad no está suficientemente representada en ese entorno. Landry y Bourhis (1997, p. 27) vinculan directamente la vitalidad de una lengua al grado de representación pública que haya adquirido, en tanto esta última simboliza el control de los mecanismos de poder, no solo político, sino también económico y cultural (asimismo en Huebner, 2006, y Franco Rodríguez, 2008).

Esta vinculación directa entre vitalidad y presencia en el PL ha sido puesta en cuestión en estudios posteriores (Blackwood, 2015; Castillo y Sáez, 2013; Vandenbroucke, 2015), ya que la primera obedece a factores más complejos. Sin embargo, resulta indudable que las medidas glotopolíticas consideran el PL como uno de los elementos de acción clave en la promoción y la normalización de las lenguas minoritarias (Brito, 2016; Lado, 2011; Marten et al., 2012).

Con el desarrollo de la disciplina de estudio del PL se han aportado nuevas perspectivas acerca de la funcionalidad del PL, incorporando nuevos cometidos; por ejemplo, Shohamy y Gorter (2009) hablan también de fines comerciales o transgresores; Spolsky (2009) incluye el objetivo de señalar la propiedad y la pertenencia; Calvi (2018), el propósito persuasivo, y Pons (2012, citando a Lapresta, 2008), destaca la importancia, en entornos de conflicto, de la función identitaria. Pons (2012) concluye que: 
Como en muchos otros aspectos de la Lingüística actual, también en el ámbito de la funcionalidad carece de sentido separar de forma discreta los posibles cometidos de un signo. Podremos describir estratos de significados que serán otorgados por los espectadores del signo en función del propósito que ellos busquen al atender al paisaje circundante (p. 81).

De hecho, se ha puesto en duda la función informativa del PL, en el sentido de que aquellos elementos que pudieran parecer referenciales (nombres de calles, información turística) "vehiculan ciertos valores e ideologías lingüísticas, como bien se ve en las zonas bilingües" (Calvi, 2018, p. 17), donde en ocasiones se han producido conflictos lingüísticos, como ha ocurrido, por ejemplo, por la determinación de la forma de los topónimos correspondientes.

El término "simbólico" ha seguido empleándose, incluso para la propia redefinición del PL: "the symbolic construction of the public space" (Ben-Rafael et al., 2006, p. 10), pero, sobre todo, en el análisis concreto, para explicar los valores añadidos y figurados que emergen del empleo de las diferentes señales. La exploración de la vertiente simbólica puede ser de especial interés, porque revela el sistema de reproducción social (Blommaert, 1999, pp. 10-11), es decir, el conjunto de agentes influyentes en el $\mathrm{PL}$, que desempeñan un papel central en la extensión de ciertas ideologías (lingüísticas o no) y, sobre todo, en su normalización, o lo que es lo mismo, en reforzar la idea de que el discurso dominante es el "normal". Es decir, refleja el poder y el prestigio $y$, en ocasiones, las discrepancias que se producen entre el discurso público y el privado, contradicciones que suelen revelar, como veremos en el apartado relativo a los usos lingüísticos, la incoherencia del paisaje en cuestión (Landry y Bourhis, 1997).

También "simbólico" es un vocablo recurrente en los trabajos que se han desarrollado en el País Vasco. Disponemos ya de numerosos estudios en diferentes territorios de la comunidad autónoma, entre los que destacamos los de Jasone Cenoz, Durk Gorter y Jokin Aiestaran en el PL de San Sebastián. Estas investigaciones han trascendido el propio objeto de estudio, de modo que sus procedimientos y conclusiones han servido igualmente para conformar la metodología y la teoría de la disciplina, de la que son referencia internacional. Son valiosos los frutos de estos trabajos previos en el País Vasco, cuyos detalles iremos viendo en contraste con el entorno bilbaíno.

Podemos anclar nuestro punto de partida en las siguientes afirmaciones: frente a un mayoritario español, el vasco es la segunda lengua más frecuente en Bilbao, pero su presencia no es tanto informativa, sino que está cargada de una potente función emblemática, ya que, a su vez, posee una fuerte impronta identitaria (Aiestaran et al., 2010, 2013; Cenoz y Gorter, 2006; Gillig, 2018; Gorter et al., 2012; Gorter y Cenoz, 2009; Järlehed, 2012; Martínez, 2016). En segundo lugar, el PL permite ver (con las limitaciones que presenta) la solidez y el estatus de las distintas variedades y, sobre todo, el efecto que las políticas lingüísticas han provocado en este contexto, lo que se hace patente si comparamos la situación de variedades con diferentes grados de intervención pública (Aiestaran et al., 2010; Cenoz y Gorter, 2006; Gorter y Cenoz, 2009; Leizaola y Egaña, 2012; Martínez, 2016; Urla y Burdick, 2018; Urrutia, 1999). En tercer lugar, el inglés manifiesta una presencia cada vez más patente como evidencia del proceso de globalización económica y cultural, sobre todo en comparación con otras lenguas (italiano, francés o alemán), que se dirigen más a los turistas, pero prácticamente las lenguas de inmigración apenas son percibidas (Cenoz y Gorter, 2006; Gorter y Cenoz, 2009; Martínez, 2016). En cuarto lugar, en lo que concierne a la percepción del PL por parte de sus usuarios, se halla una cierta aceptación tácita del statu quo lingüístico de la Comunidad Autónoma Vasca (CAV), lo que implica apoyo a las medidas de salvaguarda de la lengua minoritaria (Aiestaran et al., 2010, 2013).

Finalmente, el PL es un elemento de importancia dentro de las disposiciones glotopolíticas, en la búsqueda de la revitalización de las lenguas minoritarias (Aiestaran et al., 2013; Cenoz y Gorter, 2006; Gorter et al., 2012; Urrutia, 1999) y también del entorno económico en el que se ubican sus elementos (Cenoz y Gorter, 2009; Nunes 
et al., 2008; Onofri et al., 2010; Urla y Burdick, 2018). De hecho, los trabajos en PL han modificado algunos de los procedimientos establecidos por las autoridades responsables, con el objetivo de reconducir y encauzar las normas al respecto (Urla y Burdick, 2018).

\section{Contexto de la investigación}

Bilbao es el centro de un área funcional de gran importancia en el conjunto de la CAV y también a nivel nacional. Con más de un millón de habitantes, esa conurbación, de marcado carácter industrial, se extiende a lo largo del río Nervión, en la vertiente atlántica oriental de la península ibérica. La ciudad tiene una superficie de $41,6 \mathrm{~km}^{2}$ y un censo (de 2019) de 347083 habitantes. Su pirámide poblacional indica una evolución hacia un envejecimiento de la población y una disminución de la natalidad anual. La población inmigrante ha ido aumentando a lo largo del tiempo, de forma que ha llegado a la cifra de 29815 personas de origen extranjero (Ayuntamiento de Bilbao, s. f. 2; Eustat, 2019). Según los datos de Eustat de 2019, en Vizcaya, la mayoría (47,3 \%) de los inmigrantes extranjeros procede de América, el $25 \%$ de Europa, el 21,3\% de África, y el 6,4\%, de otros lugares.

Según los datos del V Mapa sociolingüistico 2011 (Eusko Jaularitza / Gobierno Vasco, 2014), que los recoge del Censo de Población y Viviendas de 2011, en la CAV viven más de dos millones de personas con edad superior a 5 años. De acuerdo con su competencia lingüística, se distingue entre euskaldunes, es decir, hablantes de vasco como primera o segunda lengua; euskaldunes pasivos, esto es, hablantes que pueden comprenderlo, pero no es su lengua de uso, y erdaldunes, o hablantes de español. No olvidemos que el vasco, vascuence o euskera, es una lengua no indoeuropea, por lo que es muy diferente del español o castellano. Esa distancia y su complejidad hacen que su aprendizaje sea muy costoso; a esto habría que añadir épocas de represión en su uso, que supusieron una pérdida considerable de hablantes, realidad que se está intentando resolver desde los poderes públicos.

En la Tabla 1 se precisan los datos sobre el perfil lingüístico de Bilbao en términos porcentuales y atendiendo a diferentes parámetros. Tengamos en

Tabla 1 Perfil lingüístico de Bilbao

\begin{tabular}{|c|c|c|c|c|c|}
\hline & & \multicolumn{3}{|c|}{ Competencia lingüística (\%) } & \\
\hline & & Euskaldunes & Cuasi-euskaldunes & Erdaldunes & \\
\hline \multirow[t]{8}{*}{ Territorio } & Comunidad Autónoma & 36,4 & 19,3 & 44,3 & \\
\hline & Vasca & & & & \\
\hline & Provincia de Vizcaya & 30,3 & 20,8 & 48,9 & \\
\hline & Provincia de Guipúzcoa & 52,6 & 16,8 & 30,6 & \\
\hline & Provincia de Álava & 22,9 & 19,5 & 57,6 & \\
\hline & Bilbao & 22,1 & 21,8 & 56,1 & \\
\hline & & \multicolumn{4}{|c|}{ Lengua } \\
\hline & & Euskera & Español & Las dos & Otras \\
\hline Lengua materna & Bilbao & 5 & 87,3 & 3,5 & 4,2 \\
\hline $\begin{array}{l}\text { Uso lingïístico en el } \\
\text { hogar }\end{array}$ & Bilbao & 2,9 & 91,0 & 3,8 & 2,3 \\
\hline $\begin{array}{l}\text { Actitud ante la } \\
\text { promoción del } \\
\text { euskera }\end{array}$ & Bilbao & 50,6 & 33,7 & 15,7 & \\
\hline
\end{tabular}

Fuentes: Eusko Jaularitza / Gobierno Vasco (2014), Eustat (2016). 
cuenta, no obstante, que, de acuerdo con la VI Encuesta Sociolingüistica (Gobierno Vasco, Gobierno de Navarra, 2016), las cifras cambian ligeramente: el 33,9\% de la población de 16 o más años que vive en la CAV es vascohablante, el 19,1\% es vascohablante pasivo, y el $47 \%$, castellanohablante monolingüe. También son inferiores los datos sobre la competencia en euskera en Bilbao (18,6\%, 21,1 \% y $60,3 \%$ respectivamente).

Como vemos, existen diferencias notables entre los tres territorios que constituyen la CAV. Así, según la VI Encuesta Sociolingüistica (Gobierno Vasco, Gobierno de Navarra, 2016), son vascoparlantes algo más de la mitad de Guipúzcoa, más de la cuarta parte de Vizcaya y una quinta parte de Álava. En las tres capitales de provincia (Bilbao, San SebastiánDonostia y Vitoria-Gasteiz), de carácter urbano, los porcentajes disminuyen respecto a los anteriores. Es decir, en toda la comunidad y de forma intensificada en Bilbao, se destacan quienes tienen el español como primera lengua y también quienes la tienen como única variedad. El porcentaje de euskaldunes de Bilbao está 8 puntos por debajo de la media de la provincia y 14 por debajo de la CAV, pero, siguiendo la tendencia general en el territorio, los euskaldunes de Bilbao constituyen un grupo muy joven, por encima del $60 \%$ entre los menores de 20 años, mientras que el valor más bajo es el correspondiente a los habitantes de entre 70 y 74 años (5,2 \%) (Gobierno Vasco, Gobierno de Navarra, 2016). Este rejuvenecimiento está en consonancia con el aumento de euskaldunes en más de 15 puntos en los últimos 30 años, como producto de la aplicación de medidas de política lingüística, que han intentado reconducir la tendencia a la regresión que la lengua experimentó en la primera mitad del siglo $\mathrm{xx}$, aunque la viabilidad y la vitalidad del vasco no están garantizadas de manera rotunda: sigue siendo considerado por la Unesco en situación insegura (Aiestaran et al., 2010, p. 220).

Efectivamente, desde la Ley 10/1982, del 24 de noviembre, o "Ley básica de normalización de uso del euskera” (Comunidad Autónoma del País Vasco, Parlamento Vasco, 1982), hasta nuestros días, son numerosos los planes de acción autonómicos, provinciales y locales que se han llevado a efecto, con resultados muy positivos. Destacamos dos que afectan a nuestro interés: por una parte, el "Plan general de promoción del uso del euskera" de 1998, que dio lugar al "Plan de acción para la promoción del euskera”, de 2012 (ESEP), de nivel autonómico, y, por otra parte, el "Plan de acción para la promoción del euskera en Bilbao" de 2014 (también ESEP), de nivel local. El primero tiene como objetivo

\section{[...] garantizar la normalización social del euskera y lo- grar el bilingüismo equilibrado de la sociedad vasca en la CAV, con el objetivo de avanzar en la marcha hacia el uso pleno del euskera a todos los niveles" (Consejo Asesor del Euskera, Eusko Jaurlaritza / Gobierno Vas- co, 2012, p. 11),}

y el segundo incide en tres aspectos: la adquisición, el uso y la calidad del euskera (Ayuntamiento de Bilbao, s. f. 1, p. 11).

En lo que concierne al PL, se trata de un elemento recurrente en la normativa citada y otros planes; por ejemplo, la propia Ley 10/1982 funciona como una norma que regula las cuestiones relativas al uso de las lenguas: "se exigirá de los poderes públicos la puesta en marcha de políticas relativas al paisaje lingüístico en euskera" (Urrutia, 1999, p. 137). En otros textos, como el ESEP autonómico, se incide en esta misma obligación, promoviendo, entre otras, medidas como la de "euskaldunizar el paisaje lingüístico oral y escrito en el ámbito laboral y en el del comercio" y se señala el papel destacado del PL en el proceso de normalización.

\section{Método}

El estudio que presentamos es de carácter cuantitativo y cualitativo, y forma parte de una investigación más amplia, en cuya primera parte nos deteníamos en la relación entre el PL de Bilbao y su estratificación social. ${ }^{2}$ Para ello, llevamos a cabo un trabajo

2 En Fernández Juncal (2020c). En Fernández Juncal (2019, 2020a y 2020b) analizamos dos áreas de transición entre el español y el vasco. 
de campo en tres zonas, que pueden representar los tres niveles habituales en las investigaciones sociolingüísticas: alto (A), medio (B) y bajo (C), estratificados por distribución de renta. El objetivo era caracterizarlos, observar las fluctuaciones que se producen en el tipo y las funciones de las señales en las tres zonas, y obtener así información de carácter social sobre el paisaje. En el caso actual, focalizamos nuestra atención en el empleo general del vasco y el español, por lo que analizamos esas tres zonas como un todo.

\section{Recogida de datos}

La recolección de signos se efectuó en tres secciones censales de Bilbao dentro de los barrios de Abando (A), Santutxu (B) y Bilbao la Vieja / Bilbo Zaharra (C). Para ello, se ha seguido una serie de pautas: en primer lugar, se ha elegido el signo como unidad de análisis (Backhaus, 2006; Calvi, 2018). En segundo lugar, se han excluido todas las señales que no tuvieran un cierto estatismo (por lo que quedan eliminados mensajes en atuendos o elementos del aspecto personal, señales en vehículos, etc.) o que se presentaran en otra modalidad comunicativa (sonora, exclusivamente iconográfica). Por último, se ha trabajado bajo el principio de la exhaustividad, es decir, se han registrado todos los signos visibles en las áreas acotadas, incluidos los llamados "signos parásitos" (anuncios fijados en lugares no destinados a albergarlos) (Castillo y Sáez, 2011), con la distribución zonal que se especifica en la Tabla 2.

\section{Análisis de los datos}

La información lingüística y extralingüística, proveniente de las fotografías realizadas, ha sido inventariada en una base de datos, donde se ha clasificado y codificado de acuerdo con una serie de parámetros habituales en los estudios sobre PL: autoría, fijación, función y lengua de empleo. La autoría es uno de los componentes básicos del PL (Franco Rodríguez, 2009) y permite distinguir entre textos públicos o institucionales (top-down) y textos privados (bottom-up). La fijación, por su parte, permite diferenciar entre signos permanentes, que están colocados con voluntad de continuidad, y signos de tipo caduco o efímero.

Para determinar la función hemos tomado como referencia, pero con adaptaciones, la propuesta de Pons (2012), y fijamos las funciones de los signos así: textos nominales, avisos comerciales, toponimia, señalización vial, información institucional, publicidad, información turística, conmemoración, pintadas e incisiones, y otros avisos, de tipo misceláneo, y donde se integran, por ejemplo, los de carácter político o ideológico, y también anuncios privados (compras y ventas entre particulares, búsqueda u oferta de empleo, etc.), así como manifestaciones de tipo grupal. En caso de confluencia de usos, como ocurre en ocasiones, se selecciona la función primordial.

En cuanto al tratamiento de las lenguas empleadas, nos hemos centrado en la distribución de uso de las dos lenguas oficiales y autóctonas, vasco y español, aunque dejamos constancia de la presencia de otras variedades internacionales y de lenguas desconocidas, cuyo origen no ha sido posible discernir (y que se hallan mayoritariamente en forma de pintadas o incisiones).

Para la asignación de la lengua se han tenido en consideración asimismo los nombres propios

Tabla 2 Distribución zonal de fotografías y signos

\begin{tabular}{lcccc|}
\hline & Zona A & Zona B & Zona C & Total \\
\hline Fotografías & 558 & 541 & 519 & 1618 \\
Signos & 597 & 610 & 520 & 1727 \\
Número de habitantes & 738 & 1467 & 1079 & 3284 \\
\hline
\end{tabular}


(antropónimos y topónimos fundamentalmente), en coherencia con la postura de Edelman (2009), que avisa acerca de la importancia de estos elementos en la manifestación de los usos multilingües y que nosotros también hemos constatado. Esta misma autora propone varios tipos de análisis de los nombres comerciales, uno de los cuales permite su tratamiento como cualquier otro vocablo de cualquier procedencia.

Además de la información proporcionada por los registros fotográficos, hemos mantenido conversaciones y contacto posterior con responsables del Ayuntamiento de Bilbao. Gracias a ellos, hemos accedido a datos demográficos de relevancia, pero, sobre todo, hemos podido conocer, de primera mano, los criterios y procedimientos de actuación en materia lingüística por parte de ese organismo.

\section{Resultados}

El análisis de resultados se detiene en tres aspectos: en primer lugar, en la presencia de las diferentes lenguas en el PL de Bilbao, en términos de frecuencias relativas y acumuladas, con detalle de las que poseen más implantación. En segundo lugar, y centrándonos ya en la convivencia del español y el vasco, aplicamos los parámetros de clasificación al empleo de ambas variedades, comprobando, además, la significación estadística de su distribución. Por último, nos fijamos en las microfunciones que se detectan en el paisaje, que nos permiten diferenciar entre el cumplimiento de labores informativas y aquellas que tienen funciones de tipo simbólico. En este caso, además, aportamos elementos complementarios que ratifican el reparto funcional de las dos lenguas.

Tabla 3 Distribución de uso de lenguas

\begin{tabular}{|c|c|c|c|c|c|c|}
\hline & & & \multicolumn{2}{|c|}{ Frecuencia relativa } & \multicolumn{2}{|c|}{ Frecuencia acumulada } \\
\hline & & & N. ${ }^{\circ}$ & $\%$ & N. ${ }^{\circ}$ & $\%$ \\
\hline \multirow[t]{16}{*}{ Lengua } & Español & & 880 & 51 & 1280 & 74,1 \\
\hline & & Monolingïe & 713 & $(41,3)$ & & \\
\hline & & Con inglés & 79 & & & \\
\hline & & Con otras & 88 & & & \\
\hline & Vasco & & 160 & 9,3 & 558 & 32,3 \\
\hline & & Monolingije & 138 & (8) & & \\
\hline & & Con inglés & 15 & & & \\
\hline & & Con otras & 7 & & & \\
\hline & Bilingüe vasco-español & & 190 & 11 & & \\
\hline & Mixto español-vasco & & 172 & 9,9 & & \\
\hline & & Español-vasco & 37 & & & \\
\hline & & Español-NPv & 116 & & & \\
\hline & & Vasco-español & 19 & & & \\
\hline & Inglés & & 111 & 6,4 & 211 & 12,2 \\
\hline & & Monolingïe & 93 & $(5,4)$ & & \\
\hline & & Con otras & 18 & & & \\
\hline \multicolumn{3}{|c|}{ Otras lenguas } & 20 & 1,2 & 118 & 6,8 \\
\hline \multicolumn{3}{|c|}{ Multilingije } & 39 & 2,2 & & \\
\hline \multicolumn{3}{|c|}{ Desconocida } & 155 & 9 & & \\
\hline \multicolumn{3}{|l|}{ Total } & 1727 & & 2167 & \\
\hline
\end{tabular}

NPv: nombres propios vascos. 


\section{Frecuencias en la distribución de lenguas}

Como se observa en la Tabla 3 y para cumplir nuestro objetivo, hemos detallado las formas de aparición del vasco y el español, de modo que se pueda valorar con más precisión el papel de cada una en el PL. Para ello, presentamos dos valores: la frecuencia relativa, es decir, el porcentaje de cada lengua respecto a los 1727 signos del corpus, y la frecuencia acumulada, respecto a todas las apariciones de todas las variedades (2167 casos).

Además, distinguimos entre signos bilingües (con traducción exacta entre dos lenguas) y signos mixtos (con elementos de una lengua que conviven con otra en mayor o menor proporción - alternancia, en palabras de Gorter et al., 2010-). Asimismo, se pormenorizan las combinaciones de ambas entre ellas y con otras lenguas.

En el caso de los textos mixtos, se precisa la preponderancia de una u otra, y los abundantes casos de español con nombres propios vascos $(\mathrm{NPv})$. $\mathrm{Al}$ respecto, recordemos que, según Kotze y Du Plessis (2010), el valor de este tipo de vocablos es más connotativo que denotativo, por lo que su inclusión en los textos son una decisión significativa por parte de su autor.

A la vista de los datos, se ratifica el empleo mayoritario del español monolingüe, que casi ocupa la mitad del PL. Este porcentaje aumenta hasta casi dos tercios si incluimos la aparición del español en las múltiples combinaciones con el vasco o en los textos multilingües (donde hay concurso de más de dos lenguas). Por su parte, el vasco tiene una presencia limitada cuando va aislado, pero considerablemente señalada cuando está combinado con el español o inserto en textos multilingües. Sin embargo, en los textos mixtos se corrobora la limitación de su presencia, ya que solo en algo más de una décima parte de ellos es dominante el vasco frente al español.

El inglés tiene una presencia señalada cuando aparece en signos monolingües y también si contabilizamos todos los casos de aparición, que alcanzan un 12,2\%. Las otras lenguas del paisaje (francés, alemán, latín, italiano y otras aún más minoritarias, como el árabe, el japonés, el neerlandés) representan un valor reducido tanto en su frecuencia relativa como en la acumulada.

Los datos acerca de la distribución de vasco y español, en los que nos centramos de ahora en adelante, se asemejan a los hallados por Cenoz y Gorter (2006), si tomamos en cuenta solo el orden jerárquico de los datos, pero con alguna diferencia reseñable en las frecuencias. Así, la presencia del vasco monolingüe en San Sebastián (12\%) supera en 4 puntos los datos de Bilbao (8\%) y llega a alcanzar una frecuencia acumulada por encima del $50 \%$ (casi 18 puntos más que Bilbao), lo que se puede explicar si contrastamos los datos oficiales sobre competencia y uso del vasco en ambas ciudades, muy superior en el caso de la capital guipuzcoana (17 puntos porcentuales de diferencia en número de euskaldunes). Sin embargo, esa misma investigación de Cenoz y Gorter (2006) señala un intervalo para el español mucho más amplio: frente al 41,3\% de textos monolingües en español en Bilbao, hay un $36 \%$ en San Sebastián, y ante una frecuencia acumulada del $74,1 \%$ en la primera ciudad, en la segunda se supera el $80 \%$. Podemos atribuir la aparición del español con otras variedades quizás al carácter turístico más marcado de la segunda ciudad, especialmente en la zona donde se realizó el estudio, lo que implica mayor rotulación multilingüe.

Si tenemos en cuenta la frecuencia acumulada y recuperamos los datos de la Tabla 1 sobre el perfil lingüístico de Bilbao, encontramos que la presencia del euskera en su PL está por encima de su empleo en el hogar y de su condición de lengua nativa, e incluso más representado que la competencia lingüística de los habitantes de la ciudad, pero no llega de ninguna forma a las expectativas de igualación que los planes de política lingüística animan a abordar (vid. ESEP autonómico y ESEP local). 
Usos lingüísticos y parámetros de clasificación

Podemos ahondar en el papel de cada lengua en el $\mathrm{PL}$, aplicando los criterios de clasificación de signos, cuyos resultados mostramos en la Tabla 4.

Antes de interpretar los valores de la Tabla 4, conviene señalar que hemos aplicado la prueba de ji-cuadrado $\left(\chi^{2}\right)$ a los tres parámetros que hemos especificado en aquella en relación con las variedades empleadas. Los resultados confirman la pertinencia de los criterios utilizados. En el caso de la "fijación", el grado de probabilidad es significativo al nivel $p<0,005$, y al nivel $p<0,001$ para los criterios "autoría" y "función". En cualquier caso, ambas cifras son muy elocuentes. $\mathrm{Al}$ rechazarse la hipótesis nula, se ratifica la correlación entre los criterios de clasificación del PL y la presencia del español, el vasco y sus combinaciones en Bilbao.

En línea con el corpus en su conjunto, los signos en español y vasco son mayoritariamente privados, pero hay una diferencia reseñable en lo que se refiere a la autoría: los textos en combinación bilingüe son los que más intervención pública manifiestan, como se corresponde con las líneas establecidas en las normativas ESEP local y autonómica, que algunas firmas, generalmente las de mayores dimensiones, como bancos, empresas de servicios, etc., también siguen en sus comunicaciones externas. Sin embargo, vemos cómo, en ocasiones, ese precepto, que beneficia la comunicación bilingüe, no se aplica. Puede tener que ver con el hecho de que a veces los textos bilingües aparecen como signos desmembrados (los rótulos de calles o señales en contenedores se alternan a veces en una y otra lengua), a una falta de vigilancia de la comunicación pública o al solapamiento temporal de signos de épocas previas a la normativa con los actuales.

Los textos mixtos proceden casi totalmente del sector privado, en parte porque se distancian de las normas establecidas por los organismos públicos, en parte porque son más del gusto y del uso

Tabla 4 Perfil lingüístico del PL de Bilbao de acuerdo con los criterios autoría, fijación y función

\begin{tabular}{|c|c|c|c|c|c|c|}
\hline \multicolumn{2}{|l|}{ Criterios } & \multirow{2}{*}{$\begin{array}{l}\text { Español } \\
\%\end{array}$} & \multirow{2}{*}{$\begin{array}{l}\text { Vasco } \\
\%\end{array}$} & \multirow{2}{*}{$\begin{array}{l}\text { Bilingüe } \\
\%\end{array}$} & \multirow{2}{*}{$\begin{array}{l}\text { Mixto } \\
\%\end{array}$} & \multirow{2}{*}{$\begin{array}{l}\text { Total } \\
\%\end{array}$} \\
\hline & & & & & & \\
\hline \multirow[t]{2}{*}{ Autoría } & Público & 4,9 & 18,8 & 50 & 3,5 & 11,8 \\
\hline & Privado & 95,1 & 81,2 & 50 & 96,5 & 88,2 \\
\hline \multirow[t]{2}{*}{ Fijación } & Permanente & 45,9 & 35,6 & 56,8 & 45,3 & 43,3 \\
\hline & Caduco & 54,1 & 64,4 & 43,2 & 54,7 & 56,7 \\
\hline \multirow[t]{13}{*}{ Función } & Nominal & 18,8 & 13,8 & 8,9 & 28,5 & 17,7 \\
\hline & Avisos comerciales & 38,5 & 11,3 & 21,6 & 27,3 & 28,7 \\
\hline & Toponimia & 0,8 & 3,8 & 6,9 & 1,2 & 1,6 \\
\hline & Señalización vial & 1,3 & 0,6 & 10,5 & - & 1,9 \\
\hline & Información institucional & 4,8 & 13,1 & 34,2 & 7,5 & 9,1 \\
\hline & Publicidad & 12,5 & 10,6 & 6,9 & 19,8 & 11,1 \\
\hline & Información turística & - & - & 0,5 & - & 0,3 \\
\hline & Conmemoración & 0,1 & 0,6 & - & - & 0,1 \\
\hline & Pintadas e incisiones & 7,9 & 10,6 & - & 5,2 & 14,4 \\
\hline & Otros avisos & 15,3 & 35,6 & 10,5 & 10,5 & 15,1 \\
\hline & Políticos-ideológicos & $(2,1)$ & $(26,3)$ & $(4,7)$ & $(2,9)$ & $(4,4)$ \\
\hline & Privados & $(9,9)$ & $(0,6)$ & $(0,5)$ & $(4,1)$ & $(5,8)$ \\
\hline & Manifestación grupal & $(3,3)$ & $(8,7)$ & $(5,3)$ & $(3,5)$ & $(4,9)$ \\
\hline
\end{tabular}


coloquial de los particulares, que alternan expresiones en vasco dentro de unidades en español (Gorter et al., 2012). En cualquier caso, como veremos con las funciones, el vasco es mucho menos utilizado en la comunicación privada que el español, lo cual nos remite a la conocida advertencia de Landry y Bourhis (1997):

Cuanto mayor sea la discrepancia entre el lenguaje de los signos gubernamentales y el lenguaje de los signos privados, menos coherente será el carácter del paisaje lingüístico. Desde el punto de vista sociolingüístico, la diversidad lingüística de los signos privados puede reflejar de manera más realista el carácter multilingüe de un territorio, región o aglomeración urbana concretos (p. 27).

El PL se divide en partes casi iguales en lo que concierne a la fijación de sus signos, con un ligero predominio de los de tipo pasajero, lo que confirma el dinamismo del paisaje. Son los bilingües los que ofrecen un carácter más permanente, como se corresponde con su carácter institucional, y son los escritos en lengua vasca los que muestran mayor grado de fugacidad, dato este último que explicamos por las funciones mayoritarias de uso.

\section{Funcionalidad en el paisaje lingüístico}

Retomando la Tabla 4, se observan diferencias notables en la funcionalidad de las dos lenguas y de sus combinaciones. Los textos mixtos tienen poca o nula relevancia en ámbitos formales (toponimia, información institucional, señalización vial), pero superan la media en dos ámbitos: la publicidad, cuya ductilidad le permite violentar la coherencia lingüística de los signos y, por otra parte, y de manera destacada, el uso nominal, es decir, la denominación de lugares, en los que abundan los nombres propios, muchos de origen vasco. Más de la mitad de los textos bilingües está destinada a la comunicación institucional, lo que incluye la señalización vial y la toponimia, funciones todas donde supera la media del corpus.

El español es, con mucho, la lengua más empleada en los avisos comerciales y muestra porcentajes superiores a las medias en la publicidad y los avisos personales. Se trata de cometidos de carácter informativo, donde los datos trasladados deben ser comprendidos por los posibles receptores y cumplir así dos de las máximas que proponían Spolsky y Cooper: "escriba en una lengua que conozca" y 2) "escriba en una lengua que pueda ser leída por gente que usted espera que lo lea" (1991, p. 81). ${ }^{3}$ El español está entonces ligado a funciones denotativas, situación que se ratifica con su presencia casi exclusiva en mensajes cuyo contenido afecta a cuestiones vitales ("alta tensión/peligro de muerte”, “Bomberos”, etc.).

Por el contrario, el vasco monolingüe supera a las otras modalidades lingüísticas en dos apartados de muy distinta índole: los avisos de carácter político-ideológico y las pintadas e incisiones. Se trata de dos dominios donde la tarea desempeñada es más de carácter simbólico y se responde al tercer principio de Spolsky y Cooper: "escriba un signo en su propia lengua o en una lengua con la que usted desea ser identificado" (1991, p. 81). Spolsky (2009) denomina a este fenómeno symbolic value condition, y Backhaus (2006) le atribuye una motivación política o sociocultural. Como nos recuerda Kasanga (2014), los signos de protesta son una forma de discurso, un medio para difundir las demandas, expresar sentimientos y responder a la autoridad establecida.

La dicotomía informativo/simbólico se aprecia en la especialización de las lenguas de los textos mixtos, donde se asignan ambas funciones, total o parcialmente, a lenguas diferentes, como vemos en la Figura 1, de carácter comercial, y la Figura 2, de contenido político.

A ambas señales se les podría aplicar la propuesta de Franco Rodríguez (2009) acerca de las zonas de prominencia. Así, ambas poseen una sección principal con fuerte valor simbólico, más vulnerable a cuestiones de prestigio lingüístico y presión etnolingüística, y una sección informativa, que desempeña un papel instrumental para cumplir con las

3 Las traducciones del inglés son mías. 
necesidades de comunicación efectivas con el receptor. Cuanta mayor exposición tenga una lengua minoritaria en la sección informativa, mayor será

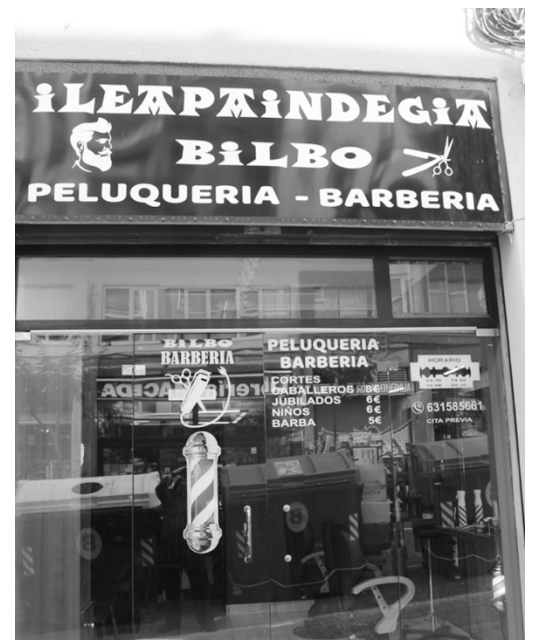

Figura 1 Signo mixto, con asignación lingüística de funciones (comercial)

Fuente: fotografía de Carmen Fernández Juncal

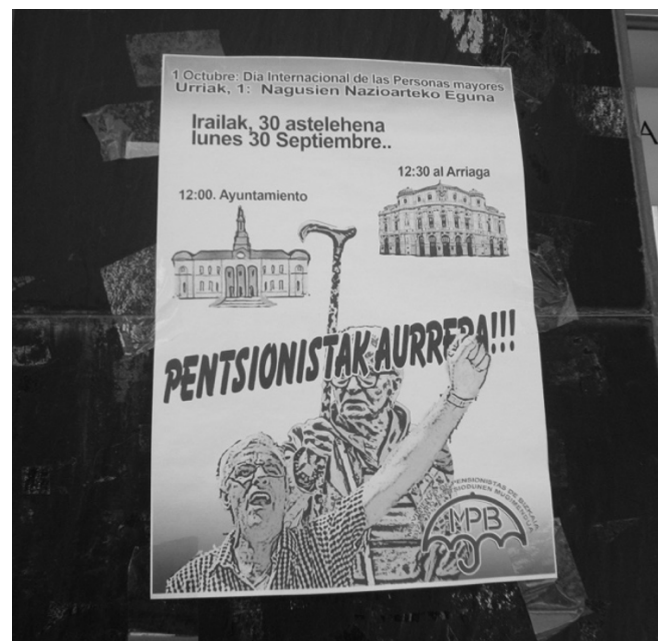

Figura 2 Signo bilingüe-mixto, con asignación lingüística de funciones (político): “Pensionistas, adelante!”

Fuente: fotografía de Carmen Fernández Juncal

su utilidad pública. En este caso, se invierten los términos: es el euskera la variedad minoritaria, con función connotativa, asociada a un fuerte valor identitario y apoyada por una política lingüística que lo promociona, mientras que el español cumple la función informativa y es simultáneamente la lengua mayoritaria.
Esta situación se puede interpretar como fase de un proceso de igualación, en el que se parte de una disparidad de uso en la zona que ha pervivido durante siglos. A la dificultad de adquisición y aprendizaje de una lengua compleja, como es el vasco, se añaden otros factores (falta de estatus oficial durante tiempo prolongado, inmigraciones, urbanización, globalización), que ralentizan el uso efectivo de esa lengua. Sin embargo, a la vista de estos ejemplos (y de otros muchos que podemos constatar en el PL de Bilbao), se puede afirmar que la labor política de poner en valor la variedad propia, es decir, el vasco, se ha conseguido, en el plano simbólico al menos (Cenoz y Gorter, 2006; Gorter et al., 2012).

Vandenbroucke (2015) emplea la noción de mercado lingüístico de Bourdieu (1991) para el caso del inglés en entornos donde no es lengua de uso: las distintas variedades se ordenan de manera jerárquica y funcionan dentro de ese mercado lingüístico como un bien o mercancía más, cuyo acceso y disfrute implica prestigio y beneficio. Pues bien, sin duda con matices, se podría extender esta situación para el vasco, que en este caso aporta interés y atractivo por sus valores de identificación grupal. Por esa razón, su presencia es especialmente notoria en los signos que se configuran como símbolos con alta carga distintiva, reivindicativa y territorial.

En esta misma línea argumentativa, señalamos dos elementos del PL de Bilbao que corroboran este valor simbólico del vasco: en primer lugar, además de una distribución funcional, se observa una distribución temático-cultural en el uso de lenguas. Así, parece que hay determinadas materias, con trasfondo cultural dispar, más proclives a ser expuestas en una y otra lengua, como se aprecia en las Figuras 3 y 4.

En segundo lugar, en muchas ocasiones destaca la presencia de la llamada "tipografía vasca", de la cual hemos mostrado dos ejemplos en las Figuras 1 y 4 , precisamente en textos escritos en vasco. De acuerdo con Järlehed (2012), su uso, extensión y significado ha ido variando con el paso del tiempo, 


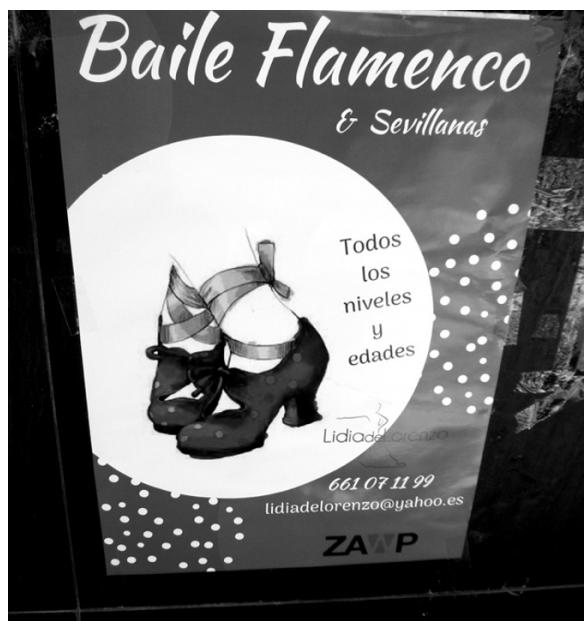

Figura 3 Texto en español

Fuente: fotografía de Carmen Fernández Juncal

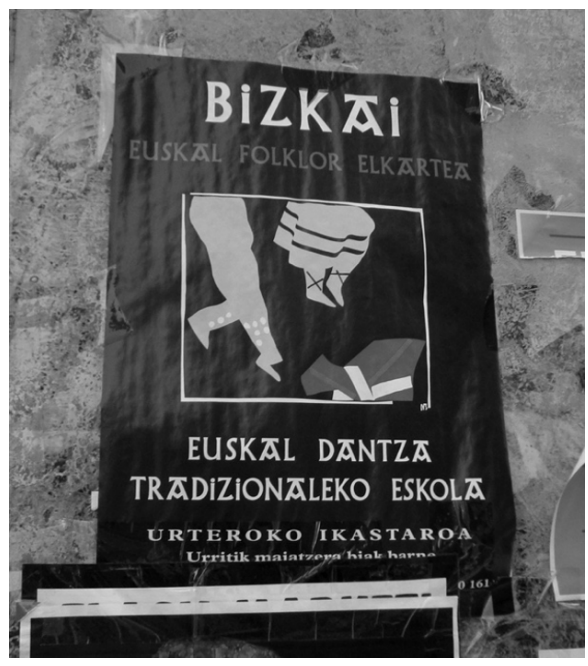

Figura 4 Texto en euskera

Fuente: fotografía de Carmen Fernández Juncal

en gran parte debido a su interacción con elementos de tipo ideológico. La letra vasca se ha venido empleando como seña de identidad de tipo social, cultural y político (Fernández Juncal, 2020a, 2020b; Gorter y Cenoz, 2015). No obstante, en otros casos se detecta que ha perdido, en cierta manera, el valor de seña política que tuvo en sus orígenes (Herrera Fernández, 2012).

\section{Conclusiones}

El País Vasco ha sido denominado "un laboratorio del multilingüismo en el espacio público" (Gorter et al., 2012, p. 161). La investigación que hemos llevado a cabo traslada el área de interés a la ciudad de Bilbao, cuyo PL no ha sido descrito ni total ni parcialmente con anterioridad, lo cual supone un avance en el inventario de usos lingüísticos en una zona de tanto interés. A partir del rastreo exhaustivo de tres secciones censales, que representan tres niveles socioeconómicos, se ha creado un corpus, cuyos elementos constitutivos han sido clasificados de acuerdo con diferentes criterios, además del lingüístico, de forma que podamos apreciar la intersección de los parámetros de tipo social con los usos lingüísticos que se producen.

Comprobamos que el perfil lingüístico de la ciudad de Bilbao muestra rasgos diferenciadores respecto a otras zonas de la CAV que ya han sido analizadas y se detecta la necesidad de determinar las diferencias sociolingüísticas dentro del mismo territorio, que van en consonancia con las políticas lingüísticas implantadas en cada lugar, como tuvimos ocasión de ratificar en trabajos previos (Fernández Juncal, 2019, 2020a, 2020b, 2020c). La puesta en marcha, desde los años ochenta, de una política lingüística robusta (Aiestaran et al., 2010) ha conseguido el desplazamiento de Bilbao desde la Primera zona sociolingüistica (menos de un $20 \%$ de la población es euskaldun) a la Segunda zona sociolingüistica (entre el 20 y el 50 \%) (Eusko Jaularitza / Gobierno Vasco, 2014).

Esta situación sociolingüística se traslada al PL, en el que el español sigue siendo la variedad de uso mayoritaria y el vasco posee una frecuencia considerable, que sobrepasa el empleo efectivo de la lengua, pero no alcanza los objetivos de paridad que se proponen desde los organismos responsables. Por consiguiente, se avanza en la controversia del PL como espejo de los usos reales, sobre todo considerando la competencia de los hablantes; sin embargo, simultáneamente, esta limitación 
está contrarrestada con la intervención lingüística pública, cuyo grado de efectividad convendría medir en el futuro, también a través de la cuantificación del PL.

Como hemos visto y en línea con trabajos precedentes, no solo en el ámbito vasco (Aiestaran et al., 2013; Cenoz y Gorter, 2006), sino también en ámbitos de contacto general de lenguas (Backhaus, 2006; Vandenbroucke, 2015), se detecta la presencia de variedades internacionales, sobre todo del inglés, cuya importancia se corrobora además en este PL, e igualmente de otras variedades (francés, italiano, alemán o latín, y otras minoritarias) con una presencia limitada en el conjunto del paisaje, que, en cualquier caso, se define como multilingüe, como se corresponde con un mundo cada vez más afectado por la globalización y la urbanización.

Centrándonos en el caso del español y el vasco, hemos comprobado la importancia de la dicotomía público/privado, oposición empleada de manera profusa en la disciplina. Se confirma que el origen público de los signos suele ser coherente y sigue pautas establecidas. Sin embargo, el ámbito privado presenta rasgos propios que convendría determinar, sobre todo, dada la importancia que posee como reflejo del uso social espontáneo (Landry y Bourhis, 1997).

En nuestra investigación, defendemos la presencia en lo privado de contradicciones internas, mayores posibilidades expresivas, oportunidad de voz para la contestación social, así como mayor gusto por la hibridación, como sucede en la comunicación oral (también, de manera indirecta, en Gillig, 2018). Si consideramos la fijación de las señales, con pocas diferencias (algo más a favor de la iniciativa pública), hay equilibrio entre los escritos con voluntad de permanencia y los que se han creado conscientes de su caducidad, lo que corrobora el carácter volátil y dinámico de los PL. Esta condición de inestabilidad de los PL precisaría de un análisis más exhaustivo de la fecha de creación de sus elementos, dato que nos ayudaría a trazar los estratos temporales que se solapan en el conjunto, limitación de este trabajo cuya resolución asumimos como reto futuro.

Si examinamos el reparto de microfunciones de una y otra lengua, asimismo se ratifica la pertinencia de aplicar a los PL los dos valores funcionales generales: lo informativo frente a lo simbólico, lo denotativo frente a lo connotativo. Sin embargo, la especialización funcional de las lenguas analizadas en este estudio, español y vasco, contraría en cierta manera la tradicional adjudicación de tareas a las lenguas mayoritarias y minoritarias, afectadas por otros factores ligados al prestigio social y ligados también a componentes de tipo identitario. Así, si consideramos la correlación entre uso y presencia en el PL, no podemos aplicar al vasco el concepto de paisaje silencioso (Castillo y Sáez, 2011), es decir, el PL que oculta los usos lingüísticos reales de la comunidad y que suele referirse a las variedades de colectivos marginados, las cuales no llegan a instalarse en el PL (Martínez, 2016). Sin embargo, no debemos olvidar que todos los procesos de políticas lingüísticas destinados a favorecer el empleo equilibrado entre lenguas de convivencia - bilingüismo, en suma - llevan incorporados medidas explícitas en el PL. Este se configura como reflejo y campo de acción en el reconocimiento igualitario de las lenguas de la comunidad, pero es necesario encontrar otras herramientas para desentrañar la realidad subyacente al discurso público. Para ello, será también preciso acudir a componentes del paisaje de carácter multimodal (iconográfico, sonoro), el primero de los cuales hemos empezado a desarrollar en este estudio.

\section{Referencias}

Aiestaran, J., Cenoz, J., y Gorter, D. (2010). Multilingual cityscapes: Perceptions and preferences of the inhabitants of the city of Donostia, San Sebastián. En E. Shohamy, E. Ben-Rafael y M. Barni (Eds.), Linguistic landscape in the city (pp. 219-234). Multilingual Matters. https://doi.org/10.21832/9781847692993-014

Aiestaran, J., Cenoz, J., y Gorter, D. (2013). Perspectivas del País Vasco: el paisaje lingüístico en Donostia, San Sebastián. Revista Internacional de Lingüistica Iberoamericana, (21), 23-38. 
Ayuntamiento de Bilbao. (s. f. 1). Plan de acción para la promoción del euskera en Bilbao (ESEP). (2014). https:// tinyurl.com/y59r33bm

Ayuntamiento de Bilbao. (s. f. 2). Servicio de datos abiertos del Ayuntamiento de Bilbao. https://www.bilbao. eus/opendata/es/inicio

Backhaus, P. (2006). Multilingualism in Tokyo: A look into the linguistic landscape. En D. Gorter (Ed.), Linguistic landscape: A new approach to multilingualism (pp. 52-66). Multilingual Matters. https://doi. org/10.21832/9781853599170-004

Ben-Rafael, E., Shohamy, E., Hasan Amara, M., y TrumperHecht, N. (2006). Linguistic landscape as symbolic construction of the public space: The case of Israel. International Journal of Multilingualism, 3(1), 7-30. https://doi.org/10.1080/14790710608668383

Blackwood, R. (2015). LL explorations and methodological challenges. Analysing France's regional languages. Linguistic Landscape, 1(1-2), 38-53. https://doi. org/10.1075/11.1.1-2.03bla

Blommaert,J.(1999).Languageideologicaldebates. DeGruyter Mouton. https://doi.org/10.1515/9783110808049

Bourdieu, P. (1991). Language and symbolic power. Harvard University Press.

Brito, A. (2016). Multilingualism and mobility: A linguistic landscape analysis of three neighbourhoods in Malmö, Sweden [M. A. Thesis]. Lund University.

Calvi, M. (2018). Español e italiano en el paisaje lingüístico de Milán. ¿Traducción, mediación o translanguaging? Lingue e Linguaggi, 25, 145-172. https://doi. org/10.1285/i22390359v25p145

Castillo Lluch, M., y Sáez Rivera, D. (2011). Introducción al paisaje lingüístico de Madrid. Lengua y Migración, 3(1), 73-88.

Castillo Lluch, M., y Sáez Rivera, D. (2013). Introducción [Sección temática: Paisajes lingüísticos en el mundo hispánico]. Revista Internacional de Lingüistica Iberoamericana, 11(21), 9-22.

Cenoz, J., y Gorter, D. (2006). Linguistic landscape and minority languages. International Journal of Multilingualism, 3(1), 67-80. https://doi. org/10.1080/14790710608668386

Cenoz, J., y Gorter, D. (2009). Language economy and linguistic landscape. En E. Shohamy y D. Gorter (Eds.), Linguistic landscape: Expanding the scenery (pp. 55-69). Routledge.

Comunidad Autónoma del País Vasco, Parlamento Vasco (1982). Ley 10/1982, básica de normalización de uso del euskera (24 de noviembre). https://www. boe.es/eli/es-pv/l/1982/11/24/10/con

Consejo Asesor del Euskera, Eusko Jaurlaritza / Gobierno Vasco (2012). Plan de acción para la promoción del euskera (ESEP). https://www.euskara.euskadi.eus/ contenidos/informacion/7041/es_2447/adjuntos/ Plan\%20ESEP_cast.pdf

Edelman, L. (2009). What's in a name? Classification of proper names by language. En E. Shohamy y D. Gorter (Eds.), Linguistic landscape: Expanding the scenery (pp. 141-154). Routledge.

Eusko Jaularitza / Gobierno Vasco. (2014). V Mapa Sociolingüistico 2011. https://www.eustat.eus/ elementos/ele0012500/v-mapa-sociolinguistico/ inf0012504_c.pdf

Eustat. (2019). Eustat - Euskal Estatistika Erakundea - Instituto Vasco de Estadística. https://www.eustat.eus

Fernández Juncal, C. (2019). Paisaje lingüístico urbano y rural: parámetros de caracterización. Cultura, lenguaje y representación, 21, 39-54. https://doi. org/10.6035/CLR.2019.21.3

Fernández Juncal, C. (2020a). El paisajelingüístico de un área urbana de transición del español y el vasco: identidades y símbolos. Bulletin Hispanique, 122, 325-344. https://doi.org/10.4000/bulletinhispanique.10906

Fernández Juncal, C. (2020b). El paisaje lingüístico de un área rural: frontera, transición y contacto. Bulletin of Hispanic Studies, 97(4), 325-347. https://doi. org/10.3828/bhs.2020.19

Fernández Juncal, C. (2020c). La estratificación social del paisaje lingüístico de Bilbao. Revista Internacional de Lingüistica Iberoamericana, 1(35), 117-140.

Franco Rodríguez, J. M. (2008). El paisaje lingüístico del condado de Los Ángeles y del condado de Miami-Dade: propuesta metodológica. Círculo de Lingüistica Aplicada a la Comunicación, (35), 3-43.

Franco Rodríguez, J. M. (2009). Interpreting the linguistic traits of linguistic landscapes as ethnolinguistic vitality: Methodological approach. Revista Electrónica de Lingüistica Aplicada, (8), 1-15.

Gillig, K. (2018). The social life of ideophones: Exploring linguistic landscaping in Basque publics [Report, The University of Texas]. https://repositories. lib.utexas.edu/bitstream/handle/2152/65775/ G I L L I G - M A S T E R S R E P O R T - 2018 . pdf ? sequence $=1 \&$ isAllowed $=y$

Gobierno Vasco, Gobierno de Navarra (2016). VIEncuesta Sociolingüistica. https://www.euskadi.eus/contenidos/ 
informacion/argitalpenak/es_6092/adjuntos/Resumen_VI_Encuesta_Sociolingü\%C3\%ADstica_ EAE_\%202016_1.pdf

Gorter, D., Aiestaran, J., y Cenoz, J. (2012). The revitalization of Basque and the linguistic landscape of Donostia-San Sebastián. En D. Gorter, H.F. Marten y L. Van Mensel (Eds.), Minority languages in the linguistic landscape (pp. 148-163). Palgrave-Macmillan. https://doi.org/10.1057/9780230360235_9

Gorter, D., y Cenoz, J. (2009). Multilingualism is all around us. Studies of the linguistic landscape. Multilingual Living Magazine, 16-18.

Gorter, D., y Cenoz, J. (2015). Translanguaging and linguistic landscapes. Linguistic Landscape, 1, (1-2), 54-74. https://doi.org/10.1075/11.1.1-2.04gor

Herrera Fernández, E. (2012). La letra vasca. Etnicidad y cultura tipográfica. Monográfica. Revista temática de diseño, (4). http://www.monografica.org/04/ Art\%C3\%ADculo/6529

Huebner, T. (2006). Bangkok's linguistic landscape: Environmental print, codemixing and language change. En D. Gorter (Ed.), Linguistic landscape: A new approach to multilingualism (pp. 31-52). Multilingual Matters. https://doi.org/10.21832/9781853599170-003

Järlehed, J. (2012). La letra vasca. Tradición inventada, nacionalismo y mercantilización cultural en el paisaje lingüístico de Euskal Herria. En T. Fernández Ulloa (Ed.), Ideology, politics and demands in spanish language, literature and film (pp. 334-357). Cambridge Scholars Publishing.

Kasanga, L. A. (2014). The linguistic landscape: Mobile signs, code choice, symbolic meaning and territoriality in the discourse of protest. International Journal of the Sociology of Language, (230), 19-44. https:// doi.org/10.1515/ijsl-2014-0025

Kotze, C. R., y Du Plessis, T. (2010). Language visibility in the Xhariep. A comparison of the linguistic landscape of three neighbouring towns. Language Matters, 41(1), 72-96. https://doi.org/10.1080/10 228195.2010 .494682

Lado, B. (2011). Linguistic landscape as a reflection of the linguistic and ideological conflict in the Valencian Community. International Journal of Multilingualism, 8(2), 135-150. https://doi.org/10.1080/14790 718.2010 .550296

Landry, R., y Bourhis, R. (1997). Linguistic landscape and ethnolinguistic vitality: An empirical study. Journal of Language and Social Psychology, 16(23), 23-49. https://doi.org/10.1177/0261927X970161002
Leizaola, A., y Egaña, M. (2012). Le paysage linguistique dans l'Eurocité basque. La signalétique routière dans une région plurilingue et transfrontalière. Analyse culturelle du paysage : le paysage comme enjeu. 135 e Congrès national des sociétés historiques et scientifiques, Neuchâtel, 2010 (pp. 98-112). http://cths. fr/ed/edition.php?id=6022

Marten, H. F., Van Mensel, L., y Gorter, D. (2012). Studying minority languages in the linguistic landscape (pp. 1-18). En D. Gorter, H.F. Marten y L. Van Mensel (Eds.), Minority languages in the linguistic landscape. Palgrave-Macmillan. https://doi. org/10.1057/9780230360235_1

Martínez, L. (2016). Dinámica de las lenguas en contacto: el paisajelingüisticodelcascoviejodeVitoria-Gasteiz [Trabajo de Grado, Universidad del País Vasco]. https:// addi.ehu.es/handle $/ 10810 / 21238$ ?show=full

Nunes, P., Onofri, L., Cenoz, J., y Gorter, D. (2008). Language diversity in urban landscapes: An econometric study. Fondazione Eni Enrico Mattei Working Papers (Paper 199). https://services.bepress.com/ feem/paper199

Onofri, L., Nunes, P., Cenoz, J., y Gorter, D. (2010). Economic preferences for language diversity: Myth or reality? An attempt to estimate the economic value of the linguistic landscape. Fondazione Eni Enrico Mattei. Working Papers (Paper 15). https://www. academia.edu/24596357/Economic_Preferences_for_Language_Diversity_A_Myth_or_Reality_ An_Attempt_to_Estimate_the_Economic_Value_ of_the_Linguistic_Landscape

Pons, L. (2012). El paisaje lingüistico de Sevilla. Lenguas y variedades en el escenario urbano hispalense. Diputación de Sevilla.

Shohamy, E., y Gorter, D. (2009). Introduction. En E. Shohamy y D. Gorter (Eds.), Linguistic landscape: Expanding the scenery (pp. 1-10). Routledge. https://doi.org/10.4324/9780203930960

Spolsky, B. (2009). Prolegomena to a sociolinguistic theory of public signage. En E. Shohamy y D. Gorter (Eds.), Linguistic landscape. Expanding the scenery (pp. 25-39). Routledge. https://doi.org/10.4324/9780203930960

Spolsky, B., y Cooper, R. L. (1991). The languages of Jerusalem. Clarendon.

Urla, J., y Burdick, C. (2018). Counting matters: Quantifying the vitality and value of Basque. International Journal of the Sociology of Language, (252), 73-96. https://doi.org/10.1515/ijsl-2018-0015

Urrutia, Í. (1999). Apuntes jurídicos sobre el pasaje lingüístico en Euskal Herria. Llengua i Dret (31), 125-182. 
Vandenbroucke, M. (2015). Language visibility, functionality and meaning across various Time Space scales in Brussels' multilingual landscapes. Journal of Mul- tilingual and Multicultural Development, 36(2), 163-181. https://doi.org/10.1080/01434632.2014 .909442

Cómo citar este artículo: Fernández-Juncal, C. (2020). Funcionalidad y convivencia del español y el vasco en el paisaje lingüístico de Bilbao. Íkala, Revista de Lenguaje y Cultura, 25(3), 713-729. https:/doi. org/10.17533/udea.ikala.v25n03a04 